\title{
Effectiveness of Task Oriented Physiotherapy Along with Conventional Physiotherapy for Patients with Stroke
}

\author{
Shahinoor Bente Khan ${ }^{1}$, Mohammad Habibur Rahman ${ }^{2}$, Md Obaidul Haque ${ }^{3}$, Ehsanur Rahman ${ }^{4}$ \\ ${ }^{1}$ Department of Physiotherapy, Centre for the Rehabilitation of the Paralysed, (CRP), Savar, Dhaka, Bangladesh \\ ${ }^{2}$ School of Science and Technology, Bangladesh Open University, Gazipur, Bangladesh \\ ${ }^{3}$ Bangladesh Health Professions Institute (BHPI), CRP, Savar, Dhaka, Bangladesh \\ ${ }^{4}$ Departmentof Physiotherapy, Bangladesh Health Professions Institute (BHPI), CRP, Savar, Dhaka, Bangladesh
}

Email address:

fnfmunna@gmail.com (S. B. Khan)

\section{To cite this article:}

Shahinoor Bente Khan, Mohammad Habibur Rahman, Md Obaidul Haque, Ehsanur Rahman. Effectiveness of Task Oriented Physiotherapy Along with Conventional Physiotherapy for Patients with Stroke. International Journal of Neurologic Physical Therapy.

Vol. 5, No. 2, 2019, pp. 37-41. doi: 10.11648/j.ijnpt.20190502.12

Received: June 24, 2019; Accepted: August 12, 2019; Published: September 16, 2019

\begin{abstract}
Background: Stroke is a disorder where brain is damaged either by blockage in the blood vessels or rupture of the blood vessels. It is the approximately number five leading cause of death. It is also the leading cause of longer period disability as well as preventable cause of disability. Objectives: To determine and compare balance, functional status and mobility before and after application of task oriented physiotherapy along with conventional physiotherapy among patients with Stroke. Methodology: Classic experimental study design was used in this study. 30 patients with stroke were randomly assigned into two groups among them 15 patients were assigned into experimental group received task oriented physiotherapy with conventional physiotherapy and another 15 into control group received only conventional physiotherapy. Total treatment sessions were twenty-four comprising of 3 sessions per week for 8 weeks. Single blinding procedure was used during data collection. Outcome measurement tools: Berg balance scale (BBS) was used to measure balance, functional independence measurement (FIM) was used to measure functional status and timed up and go test (TUG) to measure mobility. Analysis of data: Inferential statistics such as for between group analysis of BBS and FIM used Mann-Whitney U test, and within group analysis of BBS and FIM used Wilcoxon test. Besides Independent $t$ test used for between group analysis and Paired t test was done for within group analysis of TUG by using SPSS version 22. Results: It was found that balance, functional status and mobility improved both between and within group results except standing unsupported one foot in front. Conclusion: This research showed that task oriented physiotherapy along with conventional physiotherapy was more effective than only conventional physiotherapy for patients with stroke.
\end{abstract}

Keywords: Task Oriented Physiotherapy, Conventional Physiotherapy, Stroke

\section{Introduction}

A common neurological insufficiency characterized by the sudden development of a clinical sign of focal disturbance secondary to a vascular event and persists more than 24 hours known as stroke [1]. Now a day's stroke is the major familiar cause of impairments in (ADLs) activities of daily livings and it is increasing worldwide [2]. American Stroke Association (2016) stated that it is the approximately number five-leading cause of death [3]. It is also the leading cause of longer period disability. Physiotherapy is such a major component of medical science for rehabilitation of stroke patient [4]. One of the main purposes of the rehabilitative is to help patients achieve as high a level of functional independence as possible within the limits of their impairments. Task oriented physiotherapy is a treatment technique that involved the performance of repeated work; aim oriented, purposeful activities in a normal setting to facilitate individuals obtain most favorable manage strategies for alleviating association disorders and enhance the persons to lead a healthy life and improve the quality of life.

The aim of the study was to identify the effectiveness of task oriented physiotherapy along with conventional 
physiotherapy for patients with stroke.

\section{Null Hypothesis (HO)}

Task oriented physiotherapy along with conventional physiotherapy is no more effective than conventional therapy for the treatment of patients with stroke.

$H_{0}: \mu_{1}-\mu_{2}=0$ or $\mu_{1}=\mu_{2}$, where the experimental group and control group initial and final mean difference is same.

\section{Alternative Hypothesis (HA)}

Task oriented physiotherapy along with conventional physiotherapy is more effective than only conventional therapy for the treatment of patients with stroke.

$\mathrm{H}_{\mathrm{a}}: \mu_{1}-\mu_{2} \neq$ or $\mu_{1} \neq \mu_{2}$, where the experimental group and control group initial and final mean difference is not same.

\section{Methods and Materials}

The study was a quantitative evaluation of classic experimental research design. It was a single blinded study where the participants were blinded. In this study, 30 patients diagnosed with hemiplegia due to stroke were randomly assigned to balance exercise using task oriented physiotherapy group (experimental group) by 15 patient and conventional physiotherapy group (control group) of 15 patients. The present study was carried Department of physiotherapy at Centre for the Rehabilitation of the paralysed (CRP), Savar. Balance was measured usingBerg balance scale, functional status was measured by FIM scale, and Mobility was measured by TUG test.

\section{Data Collection and Analysis}

Data was collected and recorded in MS excel spread sheet 2007 and data was analyzed by using SPSS version 22. Mann Whitney $U$ test, Wilcoxon test and Independent $t$ test and paired $t$ test was admitted to find the significance between group analysis and within group analysis of BBS, FIM and TUG test.

\section{Ethical Approval}

Ethical clearance was obtained following the Bangladesh Medical Research Council (BMRC) guidelines and World Health Organization (WHO) Research guidelines. Thesis proposal was submitted for approval of the administrative bodies of ethical committee of CRP and permission was obtained from Institutional Review Board (IRB) of Bangladesh Health Profession Institute (BHPI). A pretest intervention and posttest 24 sessions of intervention was administered with each subject of both groups.

\section{Intervention Regimen Received}

Control group was given conventional physiotherapy including postural correction, maintain positioning, Bobath approach, Bobath mobilization, strengthening exercise, stretching exercise, weight bearing exercise, balance training, pelvic floor muscle strengthening exercise and also some home exercises according to patients response to treatment.

In experimental group task oriented physiotherapy was given along with conventional physiotherapy by physiotherapist who is expert in task oriented training program. The10 tasks were (1) step-ups, (2) balance board exercise, (3) kicking a ball, (4) stand up and walk, (5) obstacle course, (6) treadmill,(7) walk and carry, (8) speed walk, (9) walk backwards, and (10) stairs. Before commencing training, the subjects warmed up for 5 minutes to improve their range of motion and flexibility. Each item was practiced for 5 minutes, and 1 minute of rest time was allowed between each item (Figure 1).
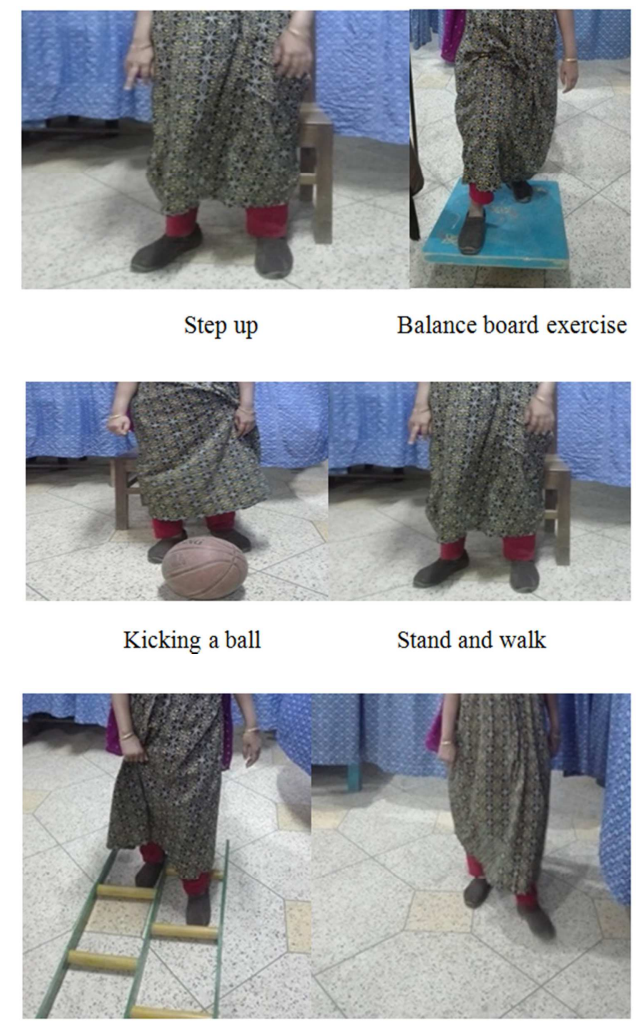

Obstacle course Walk and carry

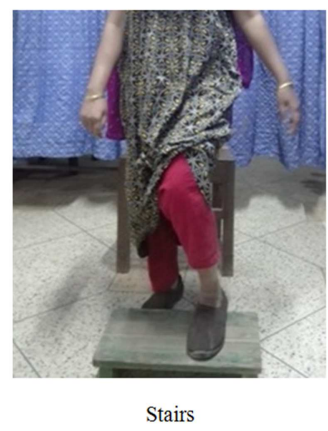

Figure 1. Description of different task oriented physiotherapy. 


\section{Result}

Table 1. Comparison of baseline characteristics of the participants.

\begin{tabular}{llll}
\hline Variable & Control group & Experimental group & P \\
\hline Age, mean (SD), years & $58.53 \pm 15.491$ & $49.60 \pm 8.903$ & 0.06 \\
Gender & Male=7 (46.7\%) Female=8 (53.3\%) & Male $=6(40 \%)$ Female=9 (60\%) & 0.52 \\
Height $(\mathrm{m})$, mean(SD) & $157.82 \pm 7.366$ & $158.50 \pm 5.581$ & 0.50 \\
Weight $(\mathrm{kg})$, mean(SD) & $63.07 \pm 9.231$ & $62.73 \pm 7.401$ & 0.43 \\
BMI (kg/m2), mean(SD) & $25.35 \pm 3.608$ & $24.90 \pm 1.927$ & 0.13 \\
TUG mean(SD), pretest & $19.87 \pm 8.015$ & $17.87 \pm 4.838$ & 0.00 \\
\hline
\end{tabular}

Table 1 compares the base line characteristics of participants between control and experimental group. In addition, two groups did not show significant differences at baseline regarding demographic characteristics instead of TUG test 0.00 . In control group the mean age $( \pm \mathrm{SD})$ of the participants was $58.53( \pm 15.491)$ years and in experimental group $49.60( \pm 8.903)$ years. In control and experimental group, male female ratio was (Male: Female=1:2). In

addition, mean height was in control group $5.19( \pm .252)$ meters and $5.24( \pm .220)$ meters in experimental group. Mean weight was $63.07( \pm 9.231) \mathrm{kg}$ in control group and $62.73( \pm 7.401) \mathrm{kg}$ in experimental group. Mean BMI was $25.33( \pm 3.586)$ in control group and $24.80( \pm 1.905)$ in experimental group. Mean $( \pm \mathrm{SD})$ pretest TUG score was in control group was $19.87 \pm 8.015$ and in contrast mean $( \pm \mathrm{SD})$ in experimental group was $17.87 \pm 4.838$.

Table 2. Mann Whitney U test for between group analysis for total BBS and FIM.

\begin{tabular}{|c|c|c|c|c|c|c|}
\hline & Category of participants & $\mathbf{N}$ & Mean of posttest & Mean rank & Mann Whitney U score & $\mathbf{P}$ \\
\hline \multirow{2}{*}{ BBS } & Experimental & 15 & 42.40 & 20.10 & \multirow{2}{*}{43} & \multirow{2}{*}{003} \\
\hline & Control & 15 & 33.07 & 10.90 & & \\
\hline \multirow{2}{*}{ FIM } & Experimental & 15 & 96.00 & 10.47 & \multirow{2}{*}{37} & \multirow{2}{*}{002} \\
\hline & Total & 30 & & & & \\
\hline
\end{tabular}

The result showed that the calculated value of $U$ is 43 for berg balance scale. From the calculated value $(U=43)$, it was clear that $U$ value between experimental and control groups had an associated probability. The level of significance is .003 which is less than 0.05 . Therefore, the result is significant for one tailed hypothesis. Since the $\mathrm{p}$ value is less than 0.05 , the result is significant and the null hypothesis (no relationship) is now rejected and the alternative hypothesis is accepted. So, it can conclude that task oriented physiotherapy along with conventional physiotherapy is more effective than only conventional therapy between group analysis of individual variables to improve balance for the patients with stroke. Mann Whitney U test score for FIM is 37. And observed $\mathrm{p}$ value is .002 which is less than 0.05 . So, we conclude that there is a significant difference between the distribution ranks in the FIM that is the exits significant difference in recalling the item between control post FIM and experimental post of FIM.

Table 3. Wiloxon test for within group analysis for total BBS and FIM.

\begin{tabular}{lllll}
\hline & Category of participants & N & Mann Whitney U score & P \\
\hline \multirow{2}{*}{ BBS } & Experimental & 15 & -3.409 & 001 \\
& Control & 15 & & 001 \\
FIM & Experimental & 15 & -3.415 & \\
& Control & 15 & & \\
& Total & 30 & & \\
\hline
\end{tabular}

The study found that the observed wilcoxon result for BBS pre and BBS post in control group was -3.409 and $p$ value was .001, significant at 5\% level of significance. For FIM in control group wilcoxon result was -3.413 and $p$ value was .001 , in experimental group was -3.415 and $\mathrm{p}$ value was.001 which is significant at 5\% level of significance. For FIM in control group wilcoxon result was -3.413 and $\mathrm{p}$ value was .001 , in experimental group was -3.415 and $p$ value was .001 which is significant at $5 \%$ level of significance.

Table 4. Between group analysis by Independent t test for in control and experimental group for TUG test.

\begin{tabular}{|c|c|c|c|c|c|}
\hline \multirow{2}{*}{ Variables } & \multirow{2}{*}{ t value } & \multirow{2}{*}{ df } & \multirow{2}{*}{ P value } & \multicolumn{2}{|c|}{ 95\% Confidence Interval } \\
\hline & & & & Lower & Upper \\
\hline Difference between control and experimental group in TUG test & 2.614 & 28 & 0.016 & 0.800 & 6.933 \\
\hline
\end{tabular}

The result showed Independent $t$ test in between group at $5 \%$ level of significant described that the calculated $t$ value is 2.614 and for $\mathrm{df}=28$, the calculated $t$ value is smaller that has an associated probability level of $.16 \%$. This means that the probability of random error being responsible for the outcome of this experiment is in 0.16 . 
Table 5. Between group analysis by Independent t test for in control and experimental group for TUG test.

\begin{tabular}{lllll}
\hline Variables & Mean & SD & Paired t & df \\
\hline TUG test Difference within control group & 4.867 & 3.378 & 5.580 & 14 \\
TUG test Difference within experimental group & 6.933 & 2.434 & 11.033 & 0.000 \\
\hline
\end{tabular}

This study found that in timed up and go test observed $t$ value was $5.580(4.867 \pm 3.378)$ in the control group at two tailed paired $\mathrm{t}$ test while this same variable for experimentalgroup observed value was $11.033(6.93 \pm 2.434) .5 \%$ level of significant at 14 (fourteen) degrees of freedom standard $t$ value was 10.500 . The observed $t$ value in experimental was more than the standard $t$ value, so null hypothesis was rejected and alternative hypothesis was accepted, that indicated that task oriented physiotherapy is effective for the patients with stroke. The observed $t$ value in control group was less than standard $t$ value that means null hypothesis was accepted and alternative hypothesis was rejected in the between group. Table showed that within group analysis of TUG test the improvement of Time up and go test was highly significant and in fact in control group $(\mathrm{p}=0.000)$ and trial group $(\mathrm{p}=0.000)$.

\section{Discussion}

The study showed significant difference in both group and following 8 weeks' task oriented physiotherapy. There was a significant improvement in balance and functional status. There are so many studies found for upper extremity and only few studies found for lower extremities. The lower extremity showed good prognosis instead of upper extremity. Some studies demonstrated that approximately $80 \%$ stroke patients survive the acute phase with walking ability and $30 \%$ to $66 \%$ facing arm disability.

In this study mobility was improved significantly. In comparison with another study proved that high intensity task oriented training can improve mobility early after stroke [5].

In this study intervention duration was 3 times per day for 45 minutes per sessions for total 8 weeks. In contrast another study conducted by Knox, Stewart and Richard (2018), showed six hour of task oriented training improve walking competency in post stroke patients [6].

Another study stated that task oriented training with cognitive behavior therapy decrease the fear of falling in chronic stroke patients [7]. It can support our study which is mobility improvement and decrease the risk of falling in stroke patients.

The present study determined the effects of task oriented physiotherapy along with conventional physiotherapy for improving balance, mobility and functional status of patients with stroke. Some studies have found that task oriented physiotherapy is effective for stroke patients. In comparison with one study conducted by Liu et al, 2019 stated that task oriented training and cognitive behavior therapy decreased fear of falling of the stroke survivors [8].

Ghazal \& Amjad (2016) stated that task oriented training improves the balance as well as balance outcome of stroke patients with diabetes. It also played an important role to reducing fall risk in diabetic population [9].
Task oriented training or task oriented physiotherapy has the capacity in case of improve balance and mobility [10]. Another study showed that task training with group therapy is a safe treatment and it is better when compared with another task training including individual task training of equal based dose. It is safe training program for self-reported mobility for the unable to walk independently stroke patients [11].

\section{Conclusion and Limitation}

\subsection{Conclusion}

Based on the result of the study was demonstrated that there was significant difference with most of the domains and some showed not significant in experimental group and control group in case of between group analysis and within group analysis. Task oriented physiotherapy helped in improving balance, mobility and functional outcome of patients with stroke. The result of the study has shown that task oriented physiotherapy along with conventional physiotherapy is superior to the conventional physiotherapy alone after twenty-four sessions of treatment for patients with stroke. From this study concluded that task oriented physiotherapy helps to improve balance, mobility and functional outcome for the patients with stroke.

\subsection{Limitation}

The study has several limitations. The sample size was very small, so the result is difficult to generalize among whole population. Researcher has taken help from one assessor for data collection purpose, it may vary result. Data was collected one clinical setting CRP Savar, it can influence the result. Sometimes treatment sessions were interrupted due to public holiday $6 \%$ participants were illiterate; it may give data error way. Therefore, the duration of the effect after the experimental intervention is unknown.

\section{Acknowledgements}

First of all, I am grateful to almighty Allah for allowing me to complete this thesis and then thanks to my parents and my supervisor to support me. I would like to thanks all physiotherapy staff and interns for helping me during data collection and treating patient for this project. I am obliged to all Physiotherapists of Neurology Unit, CRP, Savar, for their cordial support. MPT students of BHPI have supported me. I would like to express my gratitude to men and women with stroke, who gave me their valuable time and provided the information, related to my study and helped me to make my work successful. 


\section{References}

[1] Geyer JD, Gomez CR. Stroke: a practical approach. Lippincott Williams \& Wilkins; 2009.

[2] Hsieh CL, Sheu CF, Hsueh IP, Wang CH. Trunk control as an early predictor of comprehensive activities of daily living function in stroke patients. Stroke. 2002 Nov 1; 33 (11): 262630 .

[3] American Stroke association (2016), High blood pressure guidelines. Retrieved on 16th January, http://www.strokeassociation.org

[4] Davidson I, Waters K. Physiotherapists working with stroke patients: a national survey. Physiotherapy. 2000 Feb 1; 86 (2): 69-80.

[5] Outermans JC, van Peppen RP, Wittink H, Takken T, Kwakkel G. Effects of a high-intensity task-oriented training on gait performance early after stroke: a pilot study. Clinical rehabilitation. 2010 Nov; 24 (11): 979-87.

[6] Knox M, Stewart A, Richards CL. Six hours of task-oriented training optimizes walking competency post stroke: a randomized controlled trial in the public health-care system of South Africa. Clinical rehabilitation. 2018 Aug; 32 (8): $1057-$ 68.

[7] Kim BH, Lee SM, Bae YH, Yu JH, Kim TH. The effect of a task-oriented training on trunk control ability, balance and gait of stroke patients. Journal of Physical Therapy Science. 2012; 24 (6): 519-22.

[8] Liu TW, Ng GY, Chung RC, Ng SS. Decreasing fear of falling in chronic stroke survivors through cognitive behavior therapy and task-oriented training. Stroke. 2019 Jan; 50 (1): 148-54.

[9] Ghazal J, Malik AN, Amjad I. Task oriented training improves the balance outcome \& reducing fall risk in diabetic population. Pakistan journal of medical sciences. $2016 \mathrm{Jul} ; 32$ (4): 983.

[10] Yang YR, Wang RY, Lin KH, Chu MY, Chan RC. Taskoriented progressive resistance strength training improves muscle strength and functional performance in individuals with stroke. Clinical rehabilitation. 2006 Oct; 20 (10): 860-70.

[11] Kwakkel G, Kollen BJ, Wagenaar RC. Long term effects of intensity of upper and lower limb training after stroke: a randomised trial. Journal of Neurology, Neurosurgery \& Psychiatry. 2002 Apr 1; 72 (4): 473-9. 